\title{
First report of stem rot diseases of Coix lacryma-jobi caused by Fusarium incarnatum in China
}

\author{
Xiangyu Hou ${ }^{1} \cdot$ Shiyong Xie ${ }^{1} \cdot{\text { Xiaowu } \mathrm{Yu}^{2} \cdot \text { Deyi Yu}^{1} \cdot \text { Jiancheng Huang }}^{1}{ }^{\mathbb{D}}$
}

Received: 23 August 2021 / Accepted: 1 February 2022 / Published online: 16 February 2022

(c) The Author(s) under exclusive licence to Società Italiana di Patologia Vegetale (S.I.Pa.V.) 2022

Keywords Coix lacryma-jobi L $\cdot$ Stem rot · Fusarium incarnatum $\cdot$ Gene sequence analysis $\cdot$ Identification

In June 2019, a stem-rot was observed on $50 \%$ of 224 Coix lacryma-jobi L. plants of 3 cultivation farms in Pucheng county, Nanping City, Fujian Province, China. Initially, plants exhibited dark brown spots on the stem, then the whole stem base became light to dark brown including the inner tissues. Rot rapidly expanded upward until the whole plant died. Diseased tissues were surface-disinfected with $75 \%$ ethanol twice for $30 \mathrm{~s}$, rinsed with sterile water, and placed on potato dextrose agar (PDA). Colonies after 7 days at $28^{\circ} \mathrm{C}$ were round and flocculent, produced dense aerial hyphae, which were at first white, and then gradually turned orange. Macroconidia were slightly curved with 3-5 septa, 22.68 to $39.23 \mu \mathrm{m} \times 3.06$ to $5.47 \mu \mathrm{m}(\mathrm{n}=20)$. Spindle-shaped mesoconidia with 3-4 septa were abundant, 17.22 to $24.07 \mu \mathrm{m} \times 2.78$ to $4.01 \mu \mathrm{m}(\mathrm{n}=20)$. Chlamydospores were absent. The fungus was initially identified as Fusarium incarnatum (Leslie and Summerell 2006). The ITS, LSU, TUB2, EF1 and H3 gene sequences of the representative isolate PCFI-01 were amplified using primers ITS1/ITS4, NL1/NL4, TUB2a/TUB2b, EF1/EF2 and $\mathrm{H} 31 \mathrm{a} / \mathrm{H} 31 \mathrm{~b}$, respectively, and sequenced (GenBank accession Nos. ITS: MZ489433, LSU: MZ496923, TUB2: MZ497522, EF1: MZ497521, H3: MZ497523) (White et al. 1990; Vaghefi et al. 2021). BLAST analyses showed 99\% identity with $F$. incarnatum sequences in GenBank (KX965656 for ITS, MH872348 for LSU, MT895843 for TUB2, JF715935 for EF1, MH979696 for H3). Phylogenetic analysis grouped PCFI-01 with the $F$. incarnatum

\section{Jiancheng Huang}

978432655@qq.com

1 Fujian Key Laboratory for Monitoring and Integrated Management of Crop Pests, Institute of Plant

Protection, Fujian Academy of Agricultural Sciences, Fuzhou Fujian 350013, China

2 Xianyang Agricultural Technology Extension Station, Pucheng Fujian 353409, China reference strains. Pathogenicity tests were conducted on 5 healthy plants, wound-inoculated by spraying with a $10^{6}$ conidia/ml suspension of PCFI-01. Control plants were sprayed with sterile water. After 9 days, stem rot symptoms described above were observed only on inoculated plants. The fungus isolated from those plants was identified as $F$. incarnatum. To our knowledge, this is the first report of stem rot in C. lacryma-jobi caused by $F$. incarnatum.

Supplementary Information The online version contains supplementary material available at https://doi.org/10.1007/s42161-022-01049-7.

Acknowledgements The authors would like to thank the Program of Fujian Provincial Science and Technology Department (grant number 2018R1025-5) and Program of State Key Laboratory for Biology of Plant Diseases and Insect Pests (SKLOF202125) and Project of Fujian Academy of Agricultural Sciences (CXTD2021016), which funded the research.

\section{Declarations}

Conflicts of interest The authors declare that they have no conflict of interest.

\section{References}

Leslie JF, Summerell BA (2006) The Fusarium laboratory manual. Blackwell Publishing, Oxford

Vaghefi N, Shivas RG, Sharma S, Nelson SC, Pethybridge SJ (2021) Phylogeny of cercosporoid fungi (mycosphaerellaceae, mycosphaerellales) from hawaii and new york reveals novel species within the cercospora beticola complex. Mycol Progress 20:261-287

White TJ, Bruns T, Lee S, Taylor L, Shawetaylor J (1999) Amplification and direct sequencing of fungal ribosomal RNA genes for phylogenetics, in PCR Protocols: A Guide to Methods and Applications. Academic Press, New York, pp 315-322

Publisher's Note Springer Nature remains neutral with regard to jurisdictional claims in published maps and institutional affiliations. 